\title{
Feedback stabilization along a path of steady-states for 1-D semilinear heat and wave equations
}

\author{
Jean-Michel Coron and Emmanuel Trélat
}

\begin{abstract}
We report the problem of feedback stabilization along a path of steady-states, and of exact boundary controllability of semilinear one-dimensional heat and wave equations, investigated in [5], [6]. The main result is that it is possible to move from any steady-state to any other one by means of a boundary control, provided that they are in the same connected component of the set of steady-states. The proof is based on an effective feedback stabilization procedure which is efficiently implementable.
\end{abstract}

\section{INTRODUCTION}

\section{A. Semilinear one-dimensional heat equations}

In this subsection, we report on results proved in [5]. Let $L>0$ fixed and let $f: \mathbb{R} \rightarrow \mathbb{R}$ be a function of class $C^{2}$. Consider the boundary control system

$$
\left\{\begin{array}{l}
\frac{\partial y}{\partial t}=\frac{\partial^{2} y}{\partial x^{2}}+f(y) \\
y(t, 0)=0, y(t, L)=u(t)
\end{array}\right.
$$

where the state is $y(t, \cdot):[0, L] \rightarrow \mathbb{R}$ and the control is $u(t) \in \mathbb{R}$.

Concerning the global controllability problem, one of the main results [8] asserts that if $f$ is globally lipschitzian then this control system is approximately globally controllable (see also [14] for exact controllability). When $f$ is superlinear, the situation is still widely open, in particular because of possible blowing up. Indeed, if $y f(y)>0$ as $y \neq 0$, then blow-up phenomena may occur for the Cauchy problem

$$
\left\{\begin{array}{l}
\frac{\partial y}{\partial t}=\frac{\partial^{2} y}{\partial x^{2}}+f(y) \\
y(t, 0)=0, y(t, L)=0 \\
y(0, x)=y_{0}(x)
\end{array}\right.
$$

For instance, if $f(y)=y^{3}$, then, for numerous initial data, there exists $T \in(0,+\infty)$ such that the unique solution to the previous Cauchy problem is well defined on $[0, T) \times[0, L]$ and satisfies

$$
\lim _{t \rightarrow T}\|y(t, \cdot)\|_{L^{\infty}(0, L)}=+\infty
$$

see for instance [1], [11], [3], [15], [17], [20], [23] and references therein.

A natural question is then the following. Is it possible, by acting on the boundary of $[0, L]$, to avoid the blow-up

J.-M. Coron is with the Inst. Univ. France, and with the lab. AN-EDP, Université Paris-Sud, Math., UMR 8628, Bât. 425, 91405 Orsay cedex, France Jean Midhel. Coroncrath. urpstd.fr

E. Trélat is with the lab. AN-EDP, Université Paris-Sud, Math., UMR 8628, Bât. 425, 91405 Orsay cedex, France Emmanel. Trelatchath.utpsud.fr phenomenon? Actually the answer is negative in general (see [10]): for some nonlinear functions $f$ satisfying

$$
|f(y)| \sim|y| \log ^{p}(1+|y|) \quad \text { as }|y| \rightarrow+\infty,
$$

with $p>2$, and for any time $T>0$, there exist initial data which lead to blow-up before time $T$, whatever the control function $u$ is. Notice however that if

$$
|f(y)|=\mathrm{o}\left(|y| \log ^{3 / 2}(1+|y|)\right) \quad \text { as }|y| \rightarrow+\infty,
$$

then the blow-up (which could occur in the absence of control) can be avoided by means of boundary control (see [10]).

In the first case where the blow-up phenomenon cannot be compensated by means of boundary control, we propose an alternative solution. The result is that it is possible to move from any given steady-state to any other one belonging to the same connected component of the set of steady-states. More precisely, we define the notion of steady-state.

Definition 1.1: A function $y \in C^{2}([0, L])$ is a steady-state of the control system (1) if

$$
\frac{d^{2} y}{d x^{2}}+f(y)=0, y(0)=0
$$

We denote by $\mathcal{S}$ the set of steady-states, endowed with the $C^{2}$ topology.

Introduce the Banach space

$$
\begin{aligned}
& Y_{T}=\{y(t, x),(t, x) \in(0, T) \times(0, L) \mid \\
& y \in L^{2}\left(0, T, W^{2,2}(0, L)\right) \\
&\text { and } \left.\frac{\partial y}{\partial t} \in L^{2}((0, T) \times(0, L))\right\}
\end{aligned}
$$

endowed with the norm

$$
\|y\|_{Y_{T}}=\|y\|_{L^{2}\left(0, T, W^{2,2}(0, L)\right)}+\left\|\frac{\partial y}{\partial t}\right\|_{L^{2}((0, T) \times(0, L))} .
$$

Notice that $Y_{T}$ is continuously imbedded in $L^{\infty}((0, T) \times$ $(0, L))$.

The following result is proved in [5].

Theorem 1.2: Let $y_{0}$ and $y_{1}$ be two steady-states belonging to a same connected component of $\mathcal{S}$. For every neighborhood $V$ of $y_{1}$ in $H^{1}$-topology, there exists a positive real number $\varepsilon_{0}$ such that for all $\varepsilon \in\left(0, \varepsilon_{0}\right)$ there exists a control function $u \in H^{1}(0,1 / \varepsilon)$ such that the solution $y(t, x)$ in $Y_{1 / \varepsilon}$ of

$$
\left\{\begin{array}{l}
\frac{\partial y}{\partial t}=\frac{\partial^{2} y}{\partial x^{2}}+f(y) \\
y(t, 0)=0, y(t, L)=u(t) \\
y(0, x)=y_{0}(x)
\end{array}\right.
$$


satisfies $y(1 / \varepsilon, \cdot) \in V$.

Corollary 1.3: Under the assumptions of Theorem 1.2, there exist a time $T>0$ and a control function $u \in L^{2}(0, T)$ such that the solution $y(t, x)$ in $Y_{T}$ of the Cauchy-Dirichlet problem (4) satisfies $y(T, \cdot)=y_{1}(\cdot)$.

Remark 1: In [5], we give an explicit construction of the control $u$ in a feedback-type form, and of a Lyapunov functional. We stress that the procedure is effective and consists actually in solving a stabilization problem in finite dimension. Indeed in order to construct $u$ we need to compute only a finite number of quantities related to an Hilbertian expansion of the solution. The procedure has been implemented numerically and has proved to be efficient.

Remark 2: For any $T>0$ and $u \in L^{2}(0, T)$ there is at most one solution of (4) in the Banach space $Y_{T}$.

Remark 3: The corollary provides a (partial) global exact controllability result. The time needed in our proof is large, but on the other hand there are indeed cases where the time $T$ of controllability cannot be taken arbitrarily small. For instance in the case where $f(y)=-y^{3}$, any solution of (4) starting from 0 satisfies the inequality

$$
\int_{0}^{L}(L-x)^{4} y(T, x)^{2} d x \leq 8 L T,
$$

and hence if $y_{0}=0$ a minimal time is needed to reach a given $y_{1} \neq 0$. This result is similar to the one of Bamberger [13] (see also [12, Lemma 2.1]).

Remark 4: If $y_{0}$ and $y_{1}$ belong to distinct connected components of $\mathcal{S}$, then it is actually impossible to move either from $y_{0}$ to $y_{1}$ or from $y_{1}$ to $y_{0}$, whatever the time and the control are.

On the other part, in each of the following cases the set of steady-states $\mathcal{S}$ is connected:

- The function $F$ defined as

$$
F(y)=\int_{0}^{y} f(s) d s
$$

satisfies the asymptotic condition

$$
F(y) \underset{|y| \rightarrow+\infty}{\longrightarrow}+\infty .
$$

- For any $\alpha>0$ the indefinite integral

$$
\int \frac{d y}{\sqrt{\alpha-F(y)}}
$$

diverges in $-\infty$ and in $+\infty$ (if it makes sense).

- The function $f$ is odd, i.e. for any $y \in \mathbb{R}$

$$
f(-y)=-f(y) .
$$

Remark 5: The result of the corollary may be achieved directly by using repeatedly a local exact controllability theorem, see [12, Th. 4.4] or [14, Th. 3.3].

\section{B. Semilinear one-dimensional wave equations}

In this section, we announce results proved in [6]. As previously, let $L>0$ fixed and $f: \mathbb{R} \rightarrow \mathbb{R}$ be a function of class $C^{2}$. We are concerned with the exact controllability of the semilinear wave equation

$$
\left\{\begin{array}{l}
\frac{\partial^{2} y}{\partial t^{2}}=\frac{\partial^{2} y}{\partial x^{2}}+f(y), \\
y(t, 0)=0, y_{x}(t, L)=u(t), \\
y(0, \cdot)=a_{0}(\cdot), y_{t}(0, \cdot)=a_{1}(\cdot),
\end{array}\right.
$$

where the state is $\left(y(t, \cdot), y_{t}(t, \cdot)\right):[0, L] \rightarrow \mathbb{R}^{2}$ and the control is $u(t) \in \mathbb{R}$.

The question we investigate is the following. For $T>0$ large enough, given initial data $\left(a_{0}, a_{1}\right)$ and final data $\left(b_{0}, b_{1}\right)$ in a suitable Hilbert space, is it possible to construct a control $u$ steering the control system (5) from the initial state $\left(a_{0}, a_{1}\right)$ to the target $\left(b_{0}, b_{1}\right)$ within time $T$ ? Moreover, is it possible to achieve this by an explicit and efficient numerical implementation?

If $f$ is linear, the situation is well-known (see for instance [19], [22]). In the general semilinear case, the main results as to the global controllability problem, using a variant of the Hilbert Uniqueness Method and a fixed point argument, assert that if $f$ is asymptotically linear (see [24]), and more generally if $f$ is globally Lipschitzian (see [25]), then the control system (5) is globally controllable in time $T>2$, in the space $H_{(0)}^{1}(0, L) \times L^{2}(0, L)$, with controls in $L^{2}(0, T)$. The situation extends to slightly superlinear functions, or functions sharing a good sign growth condition, see [2], [18], [24], [26]. Here, $H_{(0)}^{1}(0, L)$ denotes the Banach space

$$
H_{(0)}^{1}(0, L):=\left\{y \in H^{1}(0, L) \mid y(0)=0\right\} .
$$

When $f$ is highly superlinear the situation is far more intricate, in particular because of possible blowing up, as previously for the heat equation. It is proved in [26] that if $f$ satisfies

$$
\liminf _{s \rightarrow \infty} \frac{-f(s)}{s \ln ^{p} s}>0,
$$

for $p>2$, then the system (5) is not exactly controllable in any time $T>0$. More precisely, for every $T>0$, there exist initial data $\left(a_{0}, a_{1}\right) \in H_{(0)}^{1}(0, L) \times L^{2}(0, L)$ for which the solution of (5) so that $y(0, \cdot)=a_{0}(\cdot)$ and $y_{t}(0, \cdot)=$ $a_{1}(\cdot)$ blows up in time $t<T$, for all control $u \in C([0, T])$. Hence there is no hope to get a general result on global controllability.

Definition 1.4: A function $y \in C^{2}([0, L])$ is a steady-state of the control system (5) if

$$
\frac{d^{2} y}{d x^{2}}(x)+f(y(x))=0, y(0)=0 .
$$

We denote by $\mathcal{S}$ the set of steady-states, endowed with the $C^{2}$ topology.

Let us also introduce the Banach space

$$
Y_{T}:=C^{0}\left([0, T], H^{1}(0, L) \cap C^{1}\left([0, T], L^{2}(0, L)\right) .\right.
$$

Theorem 1.5: Let $y_{0}$ and $y_{1}$ be two steady-states belonging to a same connected component of $\mathcal{S}$. For every $\delta>0$, there exists $\varepsilon_{1}>0$ so that, for every $\varepsilon \in\left(0, \varepsilon_{1}\right]$, there exists 
a control $u \in H^{2}(0,1 / \varepsilon)$ such that the solution $y$ in $Y_{1 / \varepsilon}$ of the Cauchy-Dirichlet problem

$$
\left\{\begin{array}{l}
\frac{\partial^{2} y}{\partial t^{2}}=\frac{\partial^{2} y}{\partial x^{2}}+f(y) \\
y(t, 0)=0, y_{x}(t, L)=u(t) \\
y(0, x)=y_{0}(x), y_{t}(0, x)=0
\end{array}\right.
$$

satisfies

$$
\left\|y(1 / \varepsilon, \cdot)-y_{1}(\cdot)\right\|_{H_{(0)}^{1}(0, L)}+\left\|y_{t}(1 / \varepsilon)\right\|_{L^{2}(0, L)} \leq \delta .
$$

Remark 6: In [6], we provide an explicit construction of the control $u$ in a feedback form, and of a Lyapunov functional. The procedure consists actually in solving a stabilization problem in finite dimension. Indeed in order to construct $u$, one only needs to compute a finite number of quantities related to a Riesz expansion of the solution.

Coupling Theorem 1.5 with a local controllability result yields the following corollary.

Corollary 1.6: Let $y_{0}$ and $y_{1}$ be two steady-states belonging to a same connected component of $\mathcal{S}$. There exist a time $T>0$ and a control function $u \in L^{2}(0, T)$ such that the solution $y(t, x)$ in $Y_{T}$ of the Cauchy-Dirichlet problem (7) satisfies $y(T, \cdot)=y_{1}(\cdot), y_{t}(T, \cdot)=0$.

Remark 7: The idea of stabilizing a finite dimensional part of the system is similar to the procedure achieved in the case of the heat equation. For the wave equation, it is however more challenging because of conservation properties. Moreover, one has to deal with a Riesz expansion of the solution, instead of an expansion on a Hilbertian basis of eigenfunctions.

\section{THE IDEA OF THE PROOF}

\section{A. In finite dimension}

The method is stemming from classical Lyapunov stability theory together with quasi-static deformation theory. For the sake of simplicity we explain it in finite dimension. Let us consider in $\mathbb{R}^{n}$ a general control system of the form

$$
\dot{y}(t)=g(y(t), u(t)),
$$

where $g: \mathbb{R}^{n} \times \mathbb{R}^{m} \rightarrow \mathbb{R}^{n}$ is of class $C^{1}, u(t) \in \mathcal{U}$, and $\mathcal{U}$ denotes the set of measurable essentially bounded admissible controls. Let $y_{0}, y_{1} \in \mathbb{R}^{n}$ be two equilibrium points of system (8), that is

$$
g\left(y_{i}, u_{i}\right)=0, i=0,1,
$$

for some $u_{0}, u_{1} \in \mathbb{R}^{m}$. We assume that $\left(y_{0}, u_{0}\right)$ and $\left(y_{1}, u_{1}\right)$ belong to the same connected component of the zero set of $g$ in $\mathbb{R}^{n} \times \mathbb{R}^{m}$. Our aim is to steer the system from $y_{0}$ to $y_{1}$ in some (large) time $T>0$. The method splits into four steps:

First step. Construct a $C^{1}$-path $(\bar{y}(\tau), \bar{u}(\tau))$, with $\tau \in[0,1]$, connecting $\left(y_{0}, u_{0}\right)$ to $\left(y_{1}, u_{1}\right)$ and such that

$$
\forall \tau \in[0,1] \quad g(\bar{y}(\tau), \bar{u}(\tau))=0 .
$$

Of course this path is not in general solution of system (8), but if $\varepsilon>0$ is small enough then the $C^{1}$-path $\left(y^{\varepsilon}, u^{\varepsilon}\right)$

$$
\begin{aligned}
{[0,1 / \varepsilon] } & \rightarrow \mathbb{R}^{n} \times \mathbb{R}^{m} \\
t & \mapsto\left(y^{\varepsilon}(t), u^{\varepsilon}(t)\right)=(\bar{y}(\varepsilon t), \bar{u}(\varepsilon t))
\end{aligned}
$$

is "almost" a solution of system (8). Indeed

$$
\left\|\dot{y}^{\varepsilon}-g\left(y^{\varepsilon}, u^{\varepsilon}\right)\right\|=\mathrm{O}(\varepsilon) \text { as } \varepsilon \rightarrow 0^{+} .
$$

Second step. This quasi-static trajectory is not in general stable, and thus has to be stabilized. To this aim, introduce the following change of variable:

$$
\begin{aligned}
& z(t)=y(t)-y^{\varepsilon}(t), \\
& v(t)=u(t)-u^{\varepsilon}(t),
\end{aligned}
$$

where $t \in[0,1 / \varepsilon]$. In the new variables $z, v$, the control system writes, at least if $\|z(t)\|+\|v(t)\|$ is small enough,

$\dot{z}(t)=A(\varepsilon t) z(t)+B(\varepsilon t) v(t)+\mathrm{O}\left(\|z(t)\|^{2}+\|v(t)\|^{2}+\varepsilon\right)$,

where $t \in[0,1 / \varepsilon]$, and where

$$
A(\tau)=\frac{\partial g}{\partial y}(\bar{y}(\tau), \bar{u}(\tau)),
$$

and

$$
B(\tau)=\frac{\partial g}{\partial u}(\bar{y}(\tau), \bar{u}(\tau)),
$$

with $\tau=\varepsilon t \in[0,1]$. Therefore we have to stabilize near the origin a slowly-varying in time linear control system; we refer to [16] for this classical theory.

Third step. Under mild controllability assumptions, namely

$\forall \tau \in[0,1] \quad \operatorname{rank}\left(B(\tau), A(\tau) B(\tau), \ldots, A(\tau)^{n-1} B(\tau)\right)=n$

(Kalman condition) it is actually possible to stabilize the system by pole shifting and to construct a quadratic Lyapunov function. Notice that this does not work in general if the system is not slowly-varying. So if $\varepsilon$ is small enough then using this Lyapunov function we infer that $y(1 / \varepsilon)$ belongs to some prescribed neighborhood of the target $y_{1}$. At this stage, a stabilization result is achieved.

Fourth step. If the system (8) is locally controllable near the point $y_{1}$, we conclude that it is possible to steer the system in finite time from the point $y(1 / \varepsilon)$ to the desired target $y_{1}$. Usually such a local controllability result is achieved by using an implicit function argument, after proving that the linearized system is controllable.

Remark 8: The use of quasi-static deformation for the controllability of a nonlinear partial differential control system has already been used in [4]. But note that in [4] the quasi-static trajectory $\left(y^{\varepsilon}, u^{\varepsilon}\right)$ was stable so it was not necessary to perform steps 2 and 3 . 


\section{B. Application to the heat equation}

Let $y_{0}$ and $y_{1}$ in the same connected component of $\mathcal{S}$. We construct in $\mathcal{S}$ a $C^{1}$ path $(\bar{y}(\tau, \cdot), \bar{u}(\tau)), 0 \leq \tau \leq 1$, joining $y_{0}$ to $y_{1}$. For each $i=0,1$ set

$$
\alpha_{i}=y_{i}^{\prime}(0) \text {. }
$$

Then $y_{i}(\cdot)=y^{\alpha_{i}}(\cdot), i=0,1$, where the maximal solution of

$$
\frac{d^{2} y}{d x^{2}}+f(y)=0, y(0)=0, y^{\prime}(0)=\alpha,
$$

is denoted by $y^{\alpha}(\cdot)$. Now set

$$
\bar{y}(\tau, x)=y^{(1-\tau) \alpha_{0}+\tau \alpha_{1}}(x) \text { and } \bar{u}(\tau)=\bar{y}(\tau, L),
$$

where $\tau \in[0,1]$ and $x \in[0, L]$. By construction we have

$$
\bar{y}(0, \cdot)=y_{0}(\cdot), \bar{y}(1, \cdot)=y_{1}(\cdot) \text { and } \bar{u}(0)=\bar{u}(1)=0,
$$

and thus $(\bar{y}(\tau, \cdot), \bar{u}(\tau))$ is a $C^{1}$ path in $\mathcal{S}$ connecting $y_{0}$ to $y_{1}$.

We then reduce the problem as follows.

Let $\varepsilon>0$. For every $t \in[0,1 / \varepsilon]$ and every $x \in[0, L]$, set

$$
\begin{aligned}
z(t, x) & =y(t, x)-\bar{y}(\varepsilon t, x), \\
v(t) & =u(t)-\bar{u}(\varepsilon t) .
\end{aligned}
$$

Then $z$ satisfies the initial-boundary problem

$$
\left\{\begin{array}{l}
z_{t}=z_{x x}+f^{\prime}(\bar{y}) z+z^{2} \int_{0}^{1}(1-s) f^{\prime \prime}(\bar{y}+s z) d s-\varepsilon \bar{y}_{\tau}, \\
z(t, 0)=0, z(t, L)=v(t), \\
z(0, x)=0 .
\end{array}\right.
$$

To reduce the problem to a Dirichlet-type problem, set

$$
w(t, x)=z(t, x)-\frac{x}{L} v(t),
$$

and suppose that the control $v$ is derivable. This leads to

$$
\left\{\begin{array}{l}
w_{t}=w_{x x}+f^{\prime}(\bar{y}) w+\frac{x}{L} f^{\prime}(\bar{y}) v-\frac{x}{L} v^{\prime}+r(\varepsilon, t, x), \\
w(t, 0)=w(t, L)=0 \\
w(0, x)=-\frac{x}{L} v(0)
\end{array}\right.
$$

where

$$
\begin{aligned}
& r(\varepsilon, t, x)=-\varepsilon \bar{y}_{\tau} \\
& +\left(w+\frac{x}{L} v\right)^{2} \int_{0}^{1}(1-s) f^{\prime \prime}\left(\bar{y}+s\left(w+\frac{x}{L} v\right)\right) d s .
\end{aligned}
$$

The aim is then to prove that there exist $\varepsilon$ small enough and a pair $(v, w)$ solution of (12) such that $w(1 / \varepsilon, \cdot)$ belongs to some arbitrary neighborhood of 0 in $H_{0}^{1}$-topology. To achieve this the proof consists in constructing an appropriate control function and a Lyapunov functional which stabilizes system (12) to 0 (see [5]).

The proof requires a precise spectral analysis of the problem. We introduce the one-parameter family of linear operators

$$
A(\tau)=\Delta+f^{\prime}(\bar{y}(\tau, \cdot)) I d, \tau \in[0,1],
$$

defined on $H^{2}(0, L) \cap H_{0}^{1}(0, L)$. Let $\left(e_{j}(\tau, \cdot)\right)_{j \geq 1}$ be an Hilbertian basis of $L^{2}(0, L)$ of eigenfunctions of $A(\tau)$, such that for each $j \geq 1$ and each $\tau \in[0,1]$,

$$
e_{j}(\tau, \cdot) \in H_{0}^{1}(0, L) \cap C^{2}([0, L]),
$$

and let $\left(\lambda_{j}(\tau)\right)_{j \geq 1}$ denote the corresponding eigenvalues. From the minimax principle, these eigenfunctions and eigenvalues are $C^{1}$ functions of $\tau$. Moreover for each $\tau \in[0,1]$

$$
-\infty<\cdots<\lambda_{n}(\tau)<\cdots<\lambda_{1}(\tau),
$$

and

$$
\lambda_{n}(\tau) \underset{n \rightarrow+\infty}{\longrightarrow}-\infty .
$$

From the continuity of the eigenvalues on $[0,1]$, we can define $n$ as the maximal number of eigenvalues taking at least a nonnegative value as $\tau \in[0,1]$, i.e. there exists $\eta>0$ such that

$$
\forall t \in[0,1 / \varepsilon] \quad \forall k>n \quad \lambda_{k}(\varepsilon t)<-\eta<0 .
$$

The integer $n$ can be arbitrarily large. For example if $f(y)=$ $y^{3}$ and if $y_{1}^{\prime}(0) \rightarrow+\infty$ then $n \rightarrow+\infty$.

We also set, for any $\tau \in[0,1]$ and $x \in[0, L]$,

$$
a(\tau, x)=\frac{x}{L} f^{\prime}(\bar{y}(\tau, x)) \text { and } b(x)=-\frac{x}{L} .
$$

In these notations system (12) leads to

$w_{t}(t, \cdot)=A(\varepsilon t) w(t, \cdot)+a(\varepsilon t, \cdot) v(t)+b(\cdot) v^{\prime}(t)+r(\varepsilon, t, \cdot)$.

Any solution $w(t, \cdot) \in H_{0}^{1}(0, L)$ of (16) can be expanded as series in the eigenfunctions $e_{j}(\varepsilon t, \cdot)$, convergent in $H_{0}^{1}(0, L)$,

$$
w(t, \cdot)=\sum_{j=1}^{\infty} w_{j}(t) e_{j}(\varepsilon t, \cdot) .
$$

In particular, we get, for $i=1 \ldots n$,

$$
w_{i}^{\prime}(t)=\lambda_{i}(\varepsilon t) w_{i}(t)+a_{i}(\varepsilon t) v(t)+b_{i}(\varepsilon t) v^{\prime}(t)+r_{i}^{1}(\varepsilon, t),
$$

where

$$
\begin{aligned}
r_{i}^{1}(\varepsilon, t) & =\left\langle r^{1}(\varepsilon, t, \cdot), e_{i}(\varepsilon t, \cdot)\right\rangle_{L^{2}(0, L)}, \\
a_{i}(\varepsilon t) & =\left\langle a(\varepsilon t, \cdot), e_{i}(\varepsilon t, \cdot)\right\rangle_{L^{2}(0, L)}, \\
b_{i}(\varepsilon t) & =\left\langle b(\cdot), e_{i}(\varepsilon t, \cdot)\right\rangle_{L^{2}(0, L)} .
\end{aligned}
$$

The $n$ equations (17) form a differential system controlled by $v, v^{\prime}$. Set

$$
\alpha(t)=v^{\prime}(t),
$$

and consider $v(t)$ as a state and $\alpha(t)$ as a control. Then the former finite dimensional system may be rewritten as

$$
\left\{\begin{array}{c}
v^{\prime}=\alpha, \\
w_{1}^{\prime}=\lambda_{1} w_{1}+a_{1} v+b_{1} \alpha+r_{1}^{1}, \\
\vdots \\
w_{n}^{\prime}=\lambda_{n} w_{n}+a_{n} v+b_{n} \alpha+r_{n}^{1} .
\end{array}\right.
$$


If we introduce the matrix notations

$$
\begin{gathered}
X_{1}(t)=\left(\begin{array}{c}
v(t) \\
w_{1}(t) \\
\vdots \\
w_{n}(t)
\end{array}\right), R_{1}(\varepsilon, t)=\left(\begin{array}{c}
0 \\
r_{1}^{1}(\varepsilon, t) \\
\vdots \\
r_{n}^{1}(\varepsilon, t)
\end{array}\right), \\
A_{1}(\tau)=\left(\begin{array}{cccc}
0 & 0 & \cdots & 0 \\
a_{1}(\tau) & \lambda_{1}(\tau) & \cdots & 0 \\
\vdots & \vdots & \ddots & \vdots \\
a_{n}(\tau) & 0 & \lambda_{n}(\tau)
\end{array}\right) \\
B_{1}(\tau)=\left(\begin{array}{c}
1 \\
b_{1}(\tau) \\
\vdots \\
b_{n}(\tau)
\end{array}\right),
\end{gathered}
$$

then equations (20) yield the finite dimensional linear control system

$$
X_{1}^{\prime}(t)=A_{1}(\varepsilon t) X_{1}(t)+B_{1}(\varepsilon t) \alpha(t)+R_{1}(\varepsilon, t) .
$$

We then move, by means of an appropriate feedback control, the $n$ first eigenvalues of the operator $A$, without moving the others, in order to make all eigenvalues negative.

It is easy to check that, for each $\tau \in[0,1]$ the pair $\left(A_{1}(\tau), B_{1}(\tau)\right)$ satisfies the Kalman condition, i.e.

$$
\operatorname{rank}\left(B_{1}(\tau), A_{1}(\tau) B_{1}(\tau), \ldots, A_{1}(\tau)^{n-1} B_{1}(\tau)\right)=n .
$$

Thus, for each $\tau \in[0,1]$ there exist scalars $k_{0}(\tau), \ldots, k_{n}(\tau)$ such that, if we denote

$$
K_{1}(\tau)=\left(k_{0}(\tau), \ldots, k_{n}(\tau)\right),
$$

then the matrix $A_{1}(\tau)+B_{1}(\tau) K_{1}(\tau)$ admits -1 as an eigenvalue with order $n+1$.

The remainder of the proof consists in proving, using Lyapunov functions, that the feedback control function $\alpha(t)=$ $K_{1}(\varepsilon t) X_{1}(t)$ stabilizes the complete infinite dimensional system along the path of steady-states (see [5] for details).

\section{Application to the wave equation}

The procedure is similar.

Let $\varepsilon>0$, and let $y$ denote the solution of (7) in $Y_{1 / \varepsilon}$, associated to a control $u \in H^{2}(0,1 / \varepsilon)$. We set, for all $t \in$ $[0,1 / \varepsilon]$ and $x \in[0, L]$,

$$
\begin{aligned}
z(t, x) & :=y(t, x)-\bar{y}(\varepsilon t, x), \\
u_{1}(t) & :=u(t)-\bar{u}(\varepsilon t) .
\end{aligned}
$$

Then,

$$
\left\{\begin{array}{l}
z_{t t}=z_{x x}+f^{\prime}(\bar{y}) z+z^{2} \int_{0}^{1}(1-s) f^{\prime \prime}(\bar{y}+s z) d s-\varepsilon^{2} \bar{y}_{\tau \tau} \\
z(t, 0)=0, z_{x}(t, L)=u_{1}(t) \\
z(0, x)=0, z_{t}(0, x)=-\varepsilon \bar{y}_{\tau}(0, x) .
\end{array}\right.
$$

Notice that, if the nonlinearity $f$ and the residual term $r$ were equal to zero, then, as explained previously, setting $u_{1}(t)=-\alpha z_{t}(t, L)$, the energy function

$$
t \mapsto \int_{0}^{L}\left(z_{t}(t, x)^{2}+z_{x}(t, x)^{2}\right) d t
$$

would be exponentially decreasing. This suggests to seek the control function $u_{1}(t)$ in the form

$$
u_{1}(t)=-\alpha z_{t}(t, L)+v(t),
$$

where $\alpha>0$ has to be chosen in a convenient way. Set

$$
w(t, x):=z(t, x)-\frac{x(x-L)}{L} v(t) .
$$

This leads to the system

$$
\left\{\begin{array}{l}
w_{t t}=w_{x x}+f^{\prime}(\bar{y}) w-\frac{x(x-L)}{L} v^{\prime \prime} \\
\quad+\left(\frac{x(x-L)}{L} f^{\prime}(\bar{y})+\frac{2}{L}\right) v+r(\varepsilon, t, x) \\
w(t, 0)=0, w_{x}(t, L)=-\alpha w_{t}(t, L) \\
w(0, x)=-\frac{x(x-L)}{L} v(0) \\
w_{t}(0, x)=-\varepsilon \bar{y}_{\tau}(0, x)-\frac{x(x-L)}{L} v^{\prime}(0)
\end{array}\right.
$$

where $r(\varepsilon, t, x)$ is a remainder term. The aim is to prove that, given a neighborhood $\mathcal{V}$ of $(0,0,0,0)$ in $\mathbb{R} \times \mathbb{R} \times H_{(0)}^{1}(0, L) \times$ $L^{2}(0, L)$, for $\varepsilon>0$ small enough, there exists a pair $(v, w)$ solution of (26), satisfying $v(0)=v^{\prime}(0)=0$, such that

$$
\left(v(1 / \varepsilon), v^{\prime}(1 / \varepsilon), w(1 / \varepsilon, \cdot), w_{t}(1 / \varepsilon, \cdot)\right) \in \mathcal{V} .
$$

As previously, a precise spectral analysis of the system is due. Set

$$
H:=\left\{\left(\begin{array}{l}
w^{1} \\
w^{2}
\end{array}\right) \in H^{1} \times L^{2}((0, L), \mathbb{C}) \mid w^{1}(0)=0\right\} .
$$

Introduce the one-parameter family of linear operators

$$
\widetilde{A}(\tau):=\left(\begin{array}{cc}
0 & 1 \\
A(\tau) & 0
\end{array}\right)
$$

where $A(\tau):=\triangle+f^{\prime}(\hat{y}(\tau, \cdot)) \operatorname{Id}, \tau \in[0,1]$, on the domain

$$
\begin{array}{r}
D(\tilde{A}(\tau)):=\left\{\left(\begin{array}{l}
w^{1} \\
w^{2}
\end{array}\right) \in H \mid w^{1} \in H^{2}((0, L), \mathbb{C}),\right. \\
w^{2} \in H^{1}((0, L), \mathbb{C}), \\
\left.w^{2}(0)=0, w_{x}^{1}(L)=-\alpha w^{2}(L)\right\},
\end{array}
$$

The following nontrivial lemma is crucial (see [6] for details and for a proof).

Lemma 2.1: For every $\tau \in[0,1]$, the operator $\widetilde{A}(\tau)$ has a compact resolvent in $H$, and thus its spectrum consists of isolated eigenvalues $\left(\lambda_{k}(\tau)\right)_{k \in I}$, where $I=\mathbb{Z}$ or $I=$ $\mathbb{Z} \backslash\{0\}$. There exists a Riesz basis $\left(e_{k}(\tau, \cdot)\right)_{k \in I}$ of $H$, having a dual Riesz basis $\left(f_{k}(\tau, \cdot)\right)_{k \in I}$, such that:

- $e_{k}(\tau, \cdot) \in D(\widetilde{A}(\tau))$, and $\left\|e_{k}(\tau, \cdot)\right\|_{H}=1$, for every $k \in I$ and every $\tau \in[0,1]$; 
- for every integer $k \in I$, the functions $\tau \mapsto e_{k}(\tau, \cdot)$ and $\tau \mapsto f_{k}(\tau, \cdot)$ are of class $C^{1}$ on $[0,1]$;

- each eigenvalue $\lambda_{k}(\tau)$ is geometrically simple;

- there exists an integer $n_{0} \geq 0$ so that, for every integer $k$ satisfying $|k|>n_{0}$, each eigenvalue $\lambda_{k}(\tau)$ is algebraically simple, and satisfies

$$
\lambda_{k}(\tau)=\frac{1}{2 L} \ln \frac{\alpha-1}{\alpha+1}+i \frac{k \pi}{L}+\mathrm{O}\left(\frac{1}{|k|}\right),
$$

as $|k| \rightarrow+\infty$, uniformly for $\tau \in[0,1]$;

- if $|k|>n_{0}$, then $e_{k}(\tau, \cdot)$ is an eigenfunction of $\widetilde{A}(\tau)$, associated to the (algebraically simple) eigenvalue $\lambda_{k}(\tau)$, and $f_{k}(\tau, \cdot)$ is an eigenfunction of $\widetilde{A}(\tau)^{*}$, associated to the (algebraically simple) eigenvalue $\overline{\lambda_{k}(\tau)}$;

- for every integer $k>n_{0}$ and every $\tau \in[0,1]$,

$$
\begin{gathered}
\lambda_{k}(\tau)=\overline{\lambda_{-k}(\tau)}, \\
e_{k}(\tau, \cdot)=\overline{e_{-k}(\tau, \cdot)}, \text { and } f_{k}(\tau, \cdot)=\overline{f_{-k}(\tau, \cdot)} ;
\end{gathered}
$$

- for every integer $k$ so that $|k| \leq n_{0}$, there holds

$$
\widetilde{A}(\tau) e_{k}(\tau, \cdot) \in \operatorname{Span}\left\{e_{p}(\tau, \cdot)|| p \mid \leq n_{0}\right\},
$$

and

$$
\widetilde{A}(\tau)^{*} f_{k}(\tau, \cdot) \in \operatorname{Span}\left\{f_{p}(\tau, \cdot)|| p \mid \leq n_{0}\right\} .
$$

Remark 9: To prove this lemma, we first prove the existence of a Riesz basis of $H$, consisting of generalized eigenfunctions $\left(\tilde{e}_{k}(\tau, \cdot)\right)_{k \in I}$ of $\widetilde{A}(\tau)$, associated to the eigenvalues $\left(\lambda_{k}(\tau)\right)_{k \in I}$. However, if $|k| \leq n_{0}$, then the function $\tau \mapsto \tilde{e}_{k}(\tau, \cdot)$ may fail to be of class $C^{1}$, since the corresponding eigenvalue $\lambda_{k}(\tau)$ is not necessarily algebraically simple. The proof then consists in modifying the generalized eigenfunctions $\tilde{e}_{k}(\tau, \cdot)$, for $|k| \leq n_{0}$, so as to obtain new vectors $e_{k}(\tau, \cdot),|k| \leq n_{0}$, that are $C^{1}$ functions of $\tau$, but are not necessarily generalized eigenfunctions of $\widetilde{A}(\tau)$.

Let us show how to isolate the finite dimensional unstable part of the system.

Let $\alpha>1$ so that

$$
\frac{1}{2 L} \ln \frac{\alpha-1}{\alpha+1}<-1 .
$$

Using (30), only a finite number of eigenvalues may have a nonnegative real part as $\tau \in[0,1]$. More precisely, there exists an integer $n$ so that

$\forall \tau \in[0,1], \quad \forall k \in \mathbb{Z}, \quad(|k|>n) \Rightarrow\left(\operatorname{Re}\left(\lambda_{k}(\tau)\right)<-1\right)$.

Every solution can then be expanded as series in the Riesz basis $\left(e_{j}(\varepsilon t, \cdot)\right)_{j \in I}$, convergent in $H$. As previously, we then move, by means of an appropriate feedback control, the $2 n+1$ eigenvalues $\lambda_{0}(\tau), \ldots, \lambda_{n}(\tau)$, whose real part may be nonnegative, without moving the others, so that all eigenvalues then have a negative real part. We obtain a differential system in $\mathbb{R}^{2 n+1}$ controlled by $v, v^{\prime}, v^{\prime \prime}$. As for the heat equation, we have then to check that Kalman's condition holds, which allows one to derive a feedback control stabilizing the finite dimensional system. The rest of the proof consists in showing that this control actually stabilizes the complete system (see [6]).

\section{REFERENCES}

[1] J. Bebernes and D. Eberly, Mathematical problems from combustion theory, Applied Math. Sciences, 83, Springer-Verlag, New York, 1989.

[2] P. Cannarsa, V. Komornik and P. Loreti, Well posedness and control of semilinear wave equations with iterated logarithms, ESAIM Cont. Optim. Calc. Var., 4, 1999, pp. 37-56.

[3] T. Cazenave and A. Haraux, Introduction aux problèmes d'évolution semi-linéaires, Math. \& Appl., 1, Ellipses, 1990.

[4] J. M. Coron, Local controllability of a 1-D tank containing a fluid modeled by the shallow water equations, ESAIM Control Optim. Calc. Var., 8, 2002, pp. 513-554.

[5] J.-M. Coron, E. Trélat, Global steady-state controllability of 1-D semilinear heat equations, SIAM J. Control Optim. 43, 2, 2004 , pp. 549-569.

[6] J.-M. Coron, E. Trélat, Global steady-state stabilization and controllability of 1-D semilinear wave equations, work in progress.

[7] J. I. Diaz, Obstruction and some approximate controllability results for the Burgers equation and related problems, Control of partial differential equations and applications, Lecture Notes in Pure and Appl. Math., 174, Dekker, New York, 1996, pp. 63-76.

[8] C. Fabre, J. P. Puel and E. Zuazua, Approximate controllability of the semilinear heat equation, Proc. Roy. Soc. Edinburgh Sect. A, 125, 1995, pp. 31-61.

[9] E. Fernandez-Cara, Null controllability of the semilinear heat equation, ESAIM Control Optim. Calc. Var., 2, 1997, pp. 87-103.

[10] E. Fernandez-Cara and E. Zuazua, Null and approximate controllability for weakly blowing up semilinear heat equations, Ann. Inst. $\mathrm{H}$ Poincaré Anal. Non Linéaire, 17, 5, 2000, pp. 583-616.

[11] H. Fujita, On the blowing up of solutions to the Cauchy problem for $u_{t}=\Delta u+u^{1+\alpha}$, J. Faculty Science, U. of Tokyo, 13, 1966, pp. 109124.

[12] A. Fursikov and O. Yu. Imanuvilov, On controllability of certain systems simulating a fluid flow, Flow Control, IMA, Max D. Gunzburger Editor, Springer-Verlag, 1992, pp. 149-184.

[13] J. Henry, Etude de la contrôlabilité de certaines équations paraboliques non linéaires, Thèse, Paris, 1977.

[14] O. Yu. Imanuvilov, Controllability of parabolic equations, Sb. Math., 186, 6, 1995, pp. 879-900.

[15] S. Kaplan, On the growth of solutions of quasilinear parabolic equations, Comm. Pure Appl. Math., 16, 1963, pp. 305-330.

[16] H. K. Khalil, Nonlinear Systems, Macmillan Publishing Company, New York, 1992.

[17] H. A. Levine, Some nonexistence and instability theorems for solutions of formally parabolic equations of the form $P u_{t}=-A u+F(u)$, Arch. Rat. Mech. Anal., 51, 1973, pp. 371-386.

[18] $\mathrm{L}$. Li and $\mathrm{X}$. Zhang, Exact controllability for semilinear wave equations, J. Math. Anal. Appl., 250, 2000, pp. 589-597.

[19] J. L. Lions, Exact controllability, stabilization and perturbations for distributed systems, SIAM Rev., Vol. 30, 1988, pp. 1-68.

[20] F. Merle and H. Zaag, Stability of the blow-up profile for equations of the type $u_{t}=\Delta u+|u|^{p-1} u$, Duke Math. J., 86, 1, 1997, pp. 143195.

[21] M. Reed and B. Simon, Methods of modern mathematical physics, Vol. 4, Analysis of operators, Academic Press, 1978.

[22] D. L. Russell, Controllability and stabilizability theory for linear partial differential equations: recent progress and open questions, SIAM Rev., 20, 4, 1978, pp. 639-739.

[23] H. Zaag, A remark on the energy blow-up behavior for nonlinear heat equations, Duke Math. J., 103, 3, 2000, pp. 545-555.

[24] E. Zuazua, Exact controllability for the semilinear wave equation, J. Math. Pures Appl., 69, 1990, pp. 1-31.

[25] E. Zuazua, Exact boundary controllability for the semilinear wave equation, Nonlinear partial differential equations and their applications, Collège de France seminar, Vol. X, Paris, 1987-1988, 357-391, Pitman Research Notes Math. Ser., 220, 1991.

[26] E. Zuazua, Exact controllability for semilinear wave equations in one space dimension, Ann. Inst. Henri Poincaré, Vol. 10, no. 1, 1993, pp. 109-129. 